\title{
ORIGINAL
}

\section{PERCEPCIÓN DEL ESTADO DE SALUD Y UTILIZACIÓN DE SERVICIOS SANITARIOS POR PARTE DE LAS PERSONAS INTERNAS EN UNA PRISIÓN ANDALUZA, $1999(*)$}

Eugenia Oviedo-Joekes (1), Joan Carles March Cerdà (1), Mª José Ramos Montserrat (1), Rosario Ballesta Gómez (2) y María Ángeles Prieto Rodríguez (1).

(1) Escuela Andaluza de Salud Pública

(2) Fundación Andaluza para la Atención a las Drogodependencias.

(*) El presente trabajo ha sido subvencionado por el Comisionado para la Droga, Consejería para la igualdad y el bienestar social, Junta de Andalucía.

\section{RESUMEN}

Fundamento: El estudio del estado de salud en las poblaciones penitenciarias se fundamenta en las evidencias de una salud más deteriorada que la población general y una mayor exclusión social, la cual se asocia a una peor salud general. El objetivo del trabajo es conocer la percepción del estado de salud y la utilización de los servicios sanitarios por parte de los reclusos de una cárcel de Andalucía, así como los factores asociados a dichas variables.

Métodos: Estudio descriptivo transversal. Los datos se recogieron mediante un cuestionario. El tamaño de la muestra fue de 450 reclusos de los cuales el $90,4 \%$ fueron hombres.

Resultados: El $72 \%$ de los participantes considera su salud como buena o muy buena, y el 32,7\% declaró visitar al médico una o más veces al mes. El 43,1\% de los participantes declaran padecer enfermedades crónicas, principalmente VIH $(19,1 \%)$ y hepatitis C $(18,2 \%)$ y el $40,9 \%$ que toman medicamentos. Una peor salud percibida la encontramos entre los reclusos de mayor edad, los que tienen que cumplir una condena mayor a cinco años, los que son reincidentes y los que no tienen juicios pendientes. Los que perciben que su salud se ha deteriorado en el último año presentan enfermedades crónalud se ha deteriorado en el último año presentan enfermedades crólos servicios sanitarios muestra que hacen un mayor uso de los mismos quienes tienen una condena mayor a 5 años, los que llevan menos de un año en prisión y los que toman medicamentos.

Conclusiones: Los resultados muestran la importancia de aumentar la vigilancia sobre posibles trastornos adictivos al ingreso en prisión y sobre la evolución y tratamiento de enfermedades crónicas.

Palabras clave: Prisión. Encuesta de salud. Servicios de salud.

Correspondencia:

Eugenia Oviedo Joekes

Escuela Andaluza de Salud Pública

Cuesta del Observatorio 4

Campus Universitario de Cartuja

18080 Granada

Correo electrónico: eugenia.oviedojoekes.easp@juntadeandalucia.es

\section{ABSTRACT}

\section{Perceived Health and Use of Health Care Services in Inmates of an Andalusian Prision, 1999}

Background: The study of the health condition of the populations under confinement in penitentiaries is based on the evidence of a more deteriorated health than the general population and a greater degree of social exclusion, which is associated with worse general health. This study is aimed at ascertaining how the inmates of an Andalusian penitentiary perceive their health condition and of an Andalusian penitentiary perceive their health condition and
the use made thereby of the healthcare services, as well as the factors associated with those variables.

Methods: Descriptive, cross-sectional study. The data was collected with a questionnaire. The sample size was 450 inmates, $90.4 \%$ of whom were males.

Results: Seventy-two percent of those taking part in the study considered their health to be good or very good, $32.7 \%$ stating having seen the doctor once a month or more often. A total $43.1 \%$ of the participants stated having chronic illnesses, mainly HIV (19.1\%) and hepatitis C (18.2\%); $40.9 \%$ stating that they take medication. Worse health was perceived among the older inmates, those who have to serve longer than a five-year sentence, those who are repeat offenders and those not having pending trials. Those perceiving their health to have deteriorated over the past year have chronic illnesses and take medication. The regression model for the use of healthcare services shows that they are used to a greater extent by those who are serving a longer than a 5 -year sentence, those who have been in prison for less than a year and those who take medication.

Conclusions: The results show the importance of increas the monitoring of possible addictive disorders upon entering prison and of the trend and treatment of chronic diseases.

Key words: Prisons. Health survey. Health services. 


\section{INTRODUCCIÓN}

La población penitenciara española está compuesta principalmente por varones $(92,2 \% \text { en } 2004)^{1}$, consumidores de substancias psicoactivas, con un incremento en los últimos años de personas extranjeras y reincidentes, cuyos delitos en la mayoría de los casos son contra la propiedad y la salud pública, principalmente relacionados con las drogas $^{2}$. Los problemas de salud más destacados en este medio son la tuberculosis (TBC), la infección por el virus de inmunodeficiencia humana (VIH), hepatitis C (VHC), enfermedades psiquiátricas y las drogodependencias ${ }^{3,4}$.

Además de los informes oficiales algunos estudios dan cuenta de los problemas de salud en la población penitenciara española, los cuales se centran principalmente en la alta incidencia de VIH, de TBC, así como en el análisis de conductas de riesgo de infección y transmisión de enfermedades y la adherencia a los tratamientos entre otros ${ }^{5-19}$.

El estudio del estado de salud en población penitenciaria tiene sus fundamentos en las evidencias sobre una salud más deteriorada que la población general ${ }^{4-20}$ y por estar compuesta por una alta proporción de personas que viven situaciones o procesos de exclusión social, lo cual se asocia a una peor salud general ${ }^{21-24}$. Por otra parte, existen algunos datos que indicarían que la propia situación de encarcelamiento, de permanencia en prisión, se asociaría a una desmejora en diversos indicadores de salud de las personas que cumplen condenas ${ }^{25-32}$.

Entre los indicadores de salud en prisión abordados, la salud auto-percibida y el uso de los recursos sanitarios han merecido poca atención. Dichos indicadores son relevantes de cara a conocer la apreciación interna que se tiene del estado de salud, y la atención que de hecho se recibe para el cuidado de las posibles enfermedades. Algunos trabajos han evaluado la percepción que las personas pri- vadas de libertad tienen sobre su estado de salud sugiriendo que este auto informe puede ser un robusto predictor de problemas de salud, principalmente de enfermedades crónicas $^{33,34}$. En general los estudios indican que la salud percibida en muestras de población reclusa es peor que en la población general, es decir, los presos tienden a evaluar su salud como «buena o muy buena» en menor proporción ${ }^{27,28,30,35}$. Dado que la población reclusa, como se ha indicado, presenta un mayor índice de determinadas enfermedades el uso que la misma hace de los recursos sanitarios puede ser un baremo que permita evaluar la atención y los cuidados recibidos, así como el control de la progresión de tales dolencias. Los estudios apuntan en general a que prevalecería un mayor uso de los recursos sanitarios por parte de las personas privadas de libertad ${ }^{36}$, aunque en otros se pone sobre relieve la dificultad de establecer un patrón consistente, dado que también se han encontrado con un bajo uso de los mismos ${ }^{28}$.

Para muchas personas, su ingreso en prisión puede ser la primera oportunidad de recibir atención médica ${ }^{37,38}$, convirtiéndose la prisión en un enclave estratégico para la atención y cuidado de personas que presentan enfermedades 9 . Dicha atención debería estar adaptada a las necesidades y características de la población reclusa, la cual dista mucho de la población general y ello merece que tales necesidades sean exploradas ${ }^{9-39}$.

El presente estudio tiene como objetivos conocer la percepción del estado de salud de un grupo de personas reclusas, así como los problemas de salud y la utilización de servicios sanitarios que ellas mismas refieren. Así mismo, se intentarán establecer posibles factores predictores de una peor salud percibida y de un mayor uso de los recursos sanitarios.

\section{SUJETOS Y MÉTODOS}

El estudio se llevó a cabo durante el año 1999 en una cárcel Andaluza. La muestra se 
compone de 450 participantes, lo que conforma un $35,3 \%$ de la población penitenciaria de la cárcel en el momento del estudio (aproximadamente 1.500 internos). Los participantes eran en su mayoría hombres $(90,4 \%)$ con una edad media de 32,19 años (DE: 7,56; rango 17-59), originarios principalmente de Andalucía (90\%). Los participantes se seleccionaron aleatoriamente a partir de un listado general. Las entrevistas se llevaron a cabo durante tres meses. Fueron realizadas cara a cara por cuatro personas conocedoras del ambiente carcelario formadas específicamente para este estudio. La participación en el estudio era voluntaria y confidencial, no registrándose nombre u otro código que permitiese a posteriori identificar al entrevistado. Todos los entrevistados seleccionados accedieron a participar en el estudio.

Para recoger la información se elaboró un cuestionario específico, el cual fue sometido a revisión externa por un panel de expertos en drogodependencias, instituciones penitenciarias y salud pública. Previamente a ser utilizado se realizó una prueba piloto con 60 reclusos.

El cuestionario consta de 66 preguntas cerradas y tres preguntas abiertas, de acuerdo a seis bloques temáticos: datos sociodemográficos (16 ítems), salud (9 ítems), apoyo social (10 ítems), consumo de drogas (20 ítems), sexualidad y enfermedades infecciosas (11 ítems) y un último bloque con tres preguntas abiertas correspondientes a salud percibida y propuestas de mejora. Este cuestionario ha sido utilizado en posteriores estu$\operatorname{dios}^{6}$.

\section{Análisis estadísticos}

Primeramente se realizaron análisis descriptivos, distribución de frecuencias y porcentajes, para dar cuenta de las principales características de la muestra, así como de los valores obtenidos en las variables recogidas.
Las variables de interés fueron el estado de salud percibida y la frecuencia de visitas al médico (uso de servicios sanitarios). La salud percibida, originalmente con un rango de respuestas de 5 puntos, se codificó en dos niveles: regular, malo o muy malo frente a muy bueno o bueno. Para la frecuencia de visitas al médico se utilizó como punto de corte una visita al mes, quedando esta variable con dos niveles: una vez al mes o más frente a menos de una vez al mes. Se realizaron comparaciones sobre la salud percibida y el uso de los servicios sanitarios a lo largo de las variables recogidas utilizando el estadístico Chi cuadrado. Aquellas variables que mostraron significación estadística fueron incluidas en dos modelos de regresión logística por pasos hacia atrás, para el estado de salud percibida y para la frecuencia de visitas al médico. Las variables se introdujeron por bloque (situación penitenciaria, salud, drogas, sexualidad y riesgo de infección), siendo descartadas aquellas no significativas dentro del modelo $(\mathrm{p}=0,1)$. Los análisis se realizaron con el soporte SPSS 11.0

\section{RESULTADOS}

La tabla 1 muestra las principales características de los participantes de la muestra. Para el 34,9\% del total de los internos ésta era su primera entrada en prisión. Los motivos de ingreso indican que en el 93,6\% de los casos se trataba de delitos contra la propiedad $(59,8 \%)$ o contra la salud pública $(31,1 \%)$. El 90,2\% de los internos se encontraban en situación de cumplimiento y en cuanto a la estancia en prisión el 80,9\% llevaba menos de dos años, siendo la media de años de condena de 8,66 con una moda de 3 años.

Ciento noventa y cuatro internos declararon padecer algún tipo de enfermedad crónica $(43,1 \%)$ siendo la más frecuente ser VIH positivo $(19,1 \%)$, seguida de la hepatitis C $(18,2 \%)$. Un porcentaje similar declaró haber tomado algún medicamento $(40,9 \%)$, 
Tabla 1

Variables sociodemográficas de los participantes $(n=450)$

\begin{tabular}{|c|c|c|}
\hline Variables sociodemográficas & $\mathbf{N}$ & $\%$ \\
\hline Edad media & & 32,2 \\
\hline \multicolumn{3}{|l|}{ Sexo } \\
\hline Varones & 407 & 90,4 \\
\hline Mujeres & 43 & 9,6 \\
\hline \multicolumn{3}{|l|}{ Nivel académico } \\
\hline No sabe leer ni escribir & 23 & 5,1 \\
\hline Estudios primarios incompletos & 195 & 43,3 \\
\hline Estudios primarios & 188 & 41,8 \\
\hline Estudios secundarios incompletos & 18 & 4 \\
\hline Estudios secundarios & 26 & 5,8 \\
\hline \multicolumn{3}{|l|}{ Situación socio-laboral* } \\
\hline Inactivo & 164 & 36,9 \\
\hline Precariedad laboral & 281 & 63,1 \\
\hline \multicolumn{3}{|l|}{ Situación familiar } \\
\hline Hijos & 252 & 56 \\
\hline Convivencia con pareja y/o hijos* & 419 & 93,1 \\
\hline Vivienda propia & 175 & 38,9 \\
\hline Variables penitenciarias & & \\
\hline \multicolumn{3}{|l|}{ Tipo de delito } \\
\hline Contra la propiedad & 269 & 59,8 \\
\hline Contra la salud pública & 140 & 31,1 \\
\hline Otros & 28 & 6,2 \\
\hline \multicolumn{3}{|l|}{ Situación penitenciaria } \\
\hline Preventiva & 44 & 9,8 \\
\hline Cumplimiento & 406 & 90,2 \\
\hline Tiempo medio de estancia en prisión & 1,92 & \\
\hline \multicolumn{3}{|l|}{ Salud $\dagger$} \\
\hline Buena salud percibida & 326 & 72,4 \\
\hline Padece alguna enfermedad crónica & 194 & 43,1 \\
\hline Hepatitis C & 82 & 18,2 \\
\hline $\mathrm{VIH}$ & 86 & 19,1 \\
\hline TBC & 12 & 2,7 \\
\hline
\end{tabular}

* Antes de entrar en prisión.

$\dagger$ Evaluado a través de autoinformes.

en su mayoría prescrito por el médico de la prisión, entre los que figuran psicofármacos, naltrexona, y antirretroviarales entre otros. Un 71,3\% de los reclusos se declaró consumidor de heroína, heroína mezclada con cocaína o metadona antes de entrar en pri- sión y el $52 \%$ era consumidor de cocaína o crack.

Con respecto a la percepción de salud 326 $(72,5 \%)$ internos consideraron que su salud era buena o muy buena, frente a $124(27,5 \%)$ 
Tabla 2

Variables asociadas a una peor salud percibida

\begin{tabular}{|c|c|c|}
\hline Variabes & $\begin{array}{l}\text { Recuento para cada nivel } \\
\text { de las variables } \\
(\mathbf{N}=450)\end{array}$ & $\begin{array}{c}\text { Peor salud } \\
\text { percibida* } \\
(\mathrm{N}=124) \\
\%\end{array}$ \\
\hline \multicolumn{3}{|l|}{ Tiempo en prisión ${ }^{\mathrm{b}}$} \\
\hline Menos de un año & 169 & 21,3 \\
\hline Un año & 93 & 26,9 \\
\hline Dos años & 102 & 30,4 \\
\hline Más de dos años & 86 & 37,2 \\
\hline \multicolumn{3}{|l|}{ Tiempo de condena ${ }^{a}$} \\
\hline Más de cinco años & 166 & 40,4 \\
\hline Menos de cinco años & 284 & 20,1 \\
\hline \multicolumn{3}{|l|}{ Entrada en prisión $^{\mathrm{b}}$} \\
\hline Primera entrada & 157 & 20,4 \\
\hline Reincidente & 293 & 31,4 \\
\hline Juicio pendiente $^{\text {a }}$ & 101 & 15,8 \\
\hline No & 349 & 30,9 \\
\hline Consumía drogas cuando cometió el delito ${ }^{\mathrm{c}}$ & 365 & 29,3 \\
\hline No & 85 & 20 \\
\hline \multicolumn{3}{|l|}{ Salud percibida actual en comparación con un año atrás ${ }^{a}$} \\
\hline Igual o Mejor que antes & 339 & 18,3 \\
\hline Peor que antes & 111 & 55,9 \\
\hline Enfermedad crónica $^{\text {a }}$ & 194 & 53,1 \\
\hline No & 256 & 8,2 \\
\hline Hepatitis $\mathrm{C}^{\text {a }}$ & 82 & 43,9 \\
\hline No & 368 & 23,9 \\
\hline $\mathrm{VIH}^{\mathrm{a}}$ & 86 & 70,9 \\
\hline No & 364 & 17,3 \\
\hline $\mathrm{TBC}^{\mathrm{a}}$ & 12 & 75 \\
\hline No & 438 & 26,3 \\
\hline Toma medicamentos ${ }^{\text {a }}$ & 184 & 43,5 \\
\hline & 266 & 16,5 \\
\hline Antirretrovirales ${ }^{a}$ & 40 & 70 \\
\hline No & 138 & 36,2 \\
\hline \multicolumn{3}{|l|}{ Vis a vis permitidos al mes ${ }^{a}$} \\
\hline Ninguno & 27 & 25,8 \\
\hline $\mathrm{Al}$ menos uno & 423 & 55,6 \\
\hline Fuera de la cárcel hay personas que se preocupen por $\mathrm{Ud}^{\mathrm{c}}$ & 435 & 26,9 \\
\hline No & 15 & 46,7 \\
\hline Ha consumido heroína antes de entrar en prisión ${ }^{\text {a }}$ & 223 & 33,2 \\
\hline No & 227 & 22 \\
\hline $\begin{array}{l}\text { Ha consumido Metadona prescrita antes de entrar en prisión }{ }^{a} \\
\text { No }\end{array}$ & $\begin{array}{c}66 \\
384\end{array}$ & $\begin{array}{c}36,4 \\
26\end{array}$ \\
\hline Ha consumido Metadona prescrita en el último mes ${ }^{b}$ & 84 & 38,1 \\
\hline No & 336 & 25,1 \\
\hline Tratamiento por drogas $^{\text {a }}$ & 276 & 22,8 \\
\hline No & 174 & 35,1 \\
\hline He estado en tratamiento con metadona antes de entrar en prisión ${ }^{\text {a }}$ & 123 & 36,6 \\
\hline No & 327 & 24,2 \\
\hline Querer entrar en tratamiento para drogodependencias ${ }^{\text {a }}$ & 123 & 38,4 \\
\hline No & 325 & 24,2 \\
\hline
\end{tabular}

* La salud percibida se codificó en dos niveles: regular, mala o muy mala (peor salud percibida) frente a muy buena o buena.; $a=p<0,00 ; b=p<0,05 ; c=p<0,1$.

que consideraron que ésta era regular, mala o muy mala. En la tabla 2 se muestran algunas de las variables recogidas con los valores obtenidos en sus diferentes niveles con res- pecto a un peor estado de salud percibida. Quienes son reincidentes, llevan más tiempo en prisión y tienen más años de condena perciben su salud más deteriorada en una pro- 
porción mayor que quienes han ingresado por primera vez, llevan menos tiempo en prisión y tienen menos de 5 años de condena. En cuanto al estado de salud quienes manifiestan tener una enfermedad crónica perciben su salud como mala en mayor proporción que quienes no lo hacen, presentándose la misma asociación para quienes toman medicamentos, tienen VHC o VIH. Las medidas de apoyo social no han mostrado asociaciones significativas, excepto para las visitas «vis a vis» en las que el reducido número de internos que no las tienen permitidas son los que presentan un mayor porcentaje de peor salud percibida. El consumo de drogas antes de ingresar en prisión nos muestra una asociación estadísticamente significativa sólo en el caso de la heroína y la metadona prescrita, siendo quienes las consumen los que presentan una peor percepción del estado de salud. Una menor proporción de peor salud percibida se muestra entre quienes han realizado un tratamiento por drogas, frente a los que no y entre los que desearían entrar en tratamiento frente a los que no.

El uso de los servicios sanitarios evaluado a través de la frecuencia de las visitas al médico, se reparte de la siguiente manera:

Tabla 3

Variables asociadas a una mayor utilización de los servicios sanitarios

\begin{tabular}{|c|c|}
\hline Variables & $\begin{array}{c}\text { Mayor utilización de } \\
\text { servicios sanitarios } \\
\mathrm{N}=147 \\
\end{array}$ \\
\hline \multicolumn{2}{|l|}{ Sexo ${ }^{\mathrm{c}}$} \\
\hline Hombre & 32,2 \\
\hline Mujer & 46,3 \\
\hline \multicolumn{2}{|l|}{ Tiempo en prisión ${ }^{\text {a }}$} \\
\hline Menos de un año & 42,8 \\
\hline Un año & 31,9 \\
\hline Dos años & 31,4 \\
\hline Más de dos años & 20,9 \\
\hline \multicolumn{2}{|l|}{ Entrada en prisión ${ }^{b}$} \\
\hline Primera entrada & 27 \\
\hline Reincidente & 37,1 \\
\hline \multicolumn{2}{|l|}{ Salud percibida actual en comparación con un año atrás ${ }^{a}$} \\
\hline Igual o Mejor que antes & 18,3 \\
\hline Peor que antes & 55,9 \\
\hline Toma medicamentos ${ }^{\mathrm{b}}$ & 40,3 \\
\hline No & 28,8 \\
\hline Antirretrovirales $^{\text {a }}$ & 44,1 \\
\hline No & 28,2 \\
\hline \multicolumn{2}{|l|}{ Visitas al mes $^{c}$} \\
\hline Ninguna & 36,5 \\
\hline Una o dos & 36,5 \\
\hline Más de dos & 23,5 \\
\hline Ha continuado el tratamiento en la prisión ${ }^{c}$ & 30,4 \\
\hline No & 27,9 \\
\hline Ha consumido Metadona prescrita en el último mes ${ }^{b}$ & 42,7 \\
\hline No & 31,5 \\
\hline
\end{tabular}

$\mathrm{a}=\mathrm{p}<0,00 ; \mathrm{b}=\mathrm{p}<0,05 ; \mathrm{c}=\mathrm{p}<0,1$. 
Tabla 4

Variables asociadas a una peor salud percibida mediante el ajuste de modelos de regresión logística

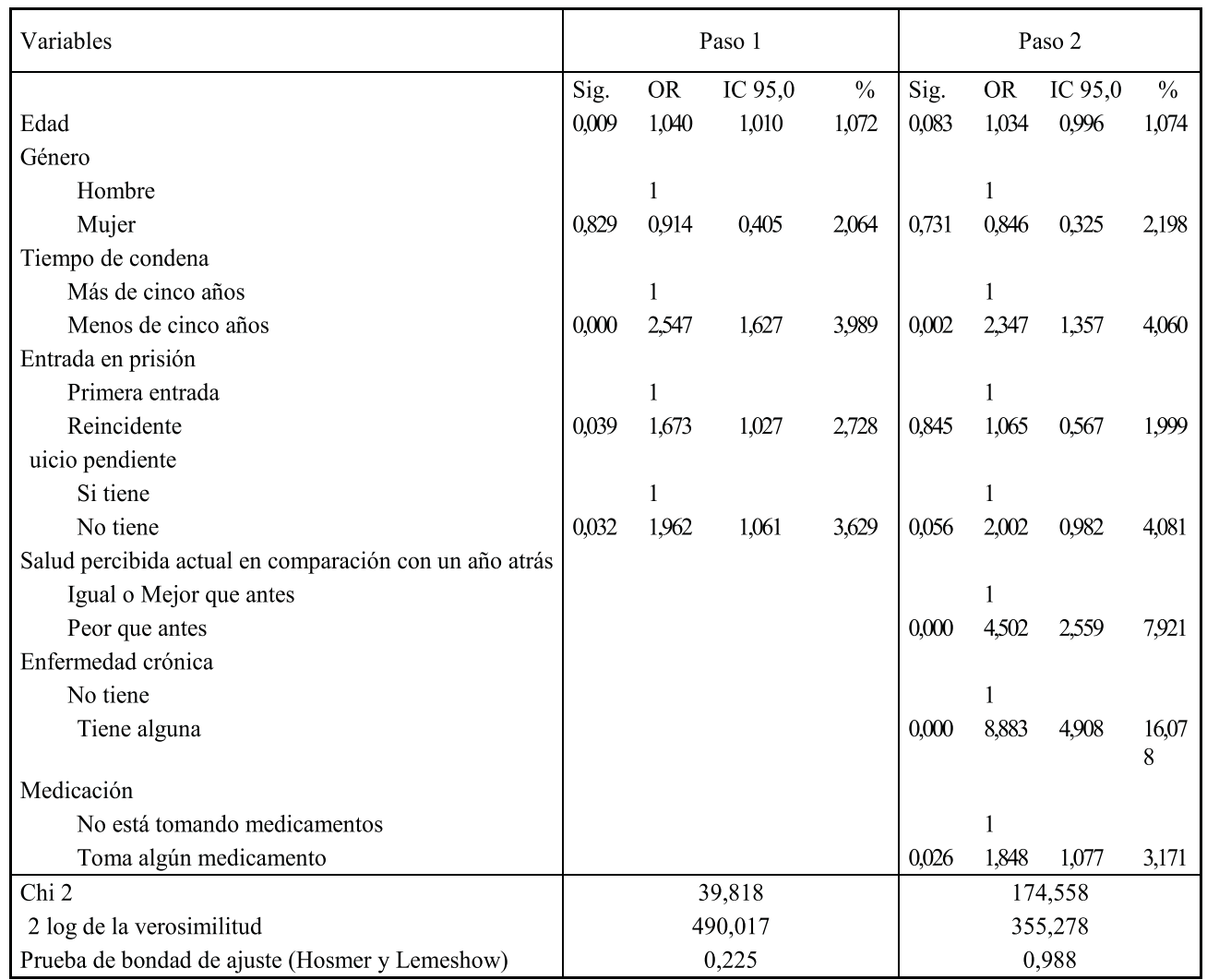

147 internos $(32,7 \%)$ declararon visitar al médico de la prisión con una frecuencia de una o más veces al mes, y $303(67,3 \%)$ con una frecuencia menor. Los motivos de consulta más frecuentes fueron cuadros agudos y revisiones de enfermedades crónicas (datos no presentados). En la tabla 3 se pueden apreciar las variables que se asocian al uso de los servicios sanitarios. Una mayor proporción del uso de los servicios sanitarios con más frecuencia la encontramos entre las mujeres, los que llevan menos tiempo en pri- sión, han ingresado por primera vez, perciben su salud actual como peor a la de hace un año, los que toman medicamentos, los que no reciben visitas y los que toman metadona prescrita.

Los modelos de regresión se muestran en las tablas 4 y 5. Para el riesgo de una peor salud percibida actual (tabla 4) no se mostraron significativas ninguna de las variables presentes en los bloques de consumo de drogas, sexualidad y riesgo de infección. Tras 
Tabla 5

Variables asociadas a un mayor uso de los servicios sanitarios mediante el ajuste de modelos de regresión logística

\begin{tabular}{|c|c|c|c|c|}
\hline \multirow{2}{*}{$\begin{array}{l}\text { Variables } \\
\text { Edad }\end{array}$} & \multirow{2}{*}{$\frac{\text { Sig. }}{0,435}$} & \multirow{2}{*}{$\begin{array}{c}\text { OR } \\
1,011\end{array}$} & \multicolumn{2}{|c|}{ IC $95,0 \%$} \\
\hline & & & 0,984 & 1,039 \\
\hline \multicolumn{5}{|l|}{ Género } \\
\hline Hombre & & 1 & & \\
\hline Mujer & 0,149 & 1,662 & 0,834 & 3,313 \\
\hline \multicolumn{5}{|l|}{ Entrada en prisión } \\
\hline Primera entrada & & 1 & & \\
\hline Reincidente & 0,009 & 1,846 & 1,166 & 2,921 \\
\hline Tiempo en prisión & 0,006 & & & \\
\hline \multicolumn{5}{|l|}{3 años o más* } \\
\hline dos años & 0,122 & 1,708 & 867 & 3,367 \\
\hline un año & 0,102 & 1,790 & 0,890 & 3,600 \\
\hline menos de un año & 0,001 & 2,924 & 1,561 & 5,477 \\
\hline \multicolumn{5}{|l|}{ Medicación } \\
\hline \multicolumn{5}{|l|}{ No está tomando medicamentos } \\
\hline Toma algún medicamento & 0,039 & 1,552 & 1,022 & 2,35 \\
\hline Chi 2 & \multicolumn{4}{|c|}{27,666} \\
\hline$-2 \log$ de la verosimilitud & \multicolumn{4}{|c|}{531,3} \\
\hline Prueba de bondad de ajuste (Hosmer y Lemeshow) & \multicolumn{4}{|c|}{0,356} \\
\hline
\end{tabular}

incluir en el modelo edad (a mayor edad peor salud percibida) y sexo como variables de control dos bloques se mostraron predictores de un peor estado de salud percibida: la situación carcelaria en cuanto al tiempo en prisión y el estado de salud. Así, un mayor riesgo de peor salud percibida lo encontramos entre los que tienen que cumplir una condena mayor a cinco años, los que son reincidentes y los que no tienen juicios pendientes. En el bloque de salud quienes perciben que la misma se ha deteriorado en el último año tienen más riesgo y, por el contrario, quienes no presentan enfermedades crónicas o no toman medicamentos tienen menos riesgo de declarar una peor salud percibida. El modelo de regresión para el riesgo de un mayor uso de los servicios sanitarios muestra que quienes tienen una condena mayor a 5 años, aquellos que llevan menos de un año en prisión y los que toman medicamentos tienen mayor riesgo. En ninguno de los modelos ha sido significativa la prueba de
Hosmer y Lemeshow, lo cual indica que los datos se ajustan a los mismos.

\section{DISCUSIÓN}

El presente estudio nos ha permitido conocer el estado de salud percibida por parte de los reclusos de una penitenciaría andaluza, así como el uso que los mismos hacen de los recursos sanitarios disponibles. También hemos podido analizar algunas de las variables asociadas a una peor percepción de la salud y a un mayor uso de los servicios sanitarios. No obstante, dado que es un diseño transversal no se pueden establecer relaciones de causa y efecto, por lo cual hablamos de asociaciones. Así mismo, puesto que el estudio se ha realizado en una sola cárcel corresponde ser cautos a la hora de extrapolar los resultados a otras instituciones penitenciarias.

Existen evidencias suficientes de que la salud percibida es un buen indicador de 
salud ya que se asocia a la mortalidad, a la capacidad funcional, y a la utilización de servicios sanitarios ${ }^{17,33,40}$. Un importante grupo de reclusos consideran su salud como buena o muy buena $(72 \%)$, porcentaje similar al obtenido en población penitenciaria en otros países ${ }^{28,35}$, e inferior al $80,8 \%$ de la población general española de entre 25 y 44 años ${ }^{41}$, en todos los casos evaluados de la misma manera.

Uno de los factores asociados a la salud percibida por los participantes ha sido la edad, resultando que a mayor edad existe peor percepción de la salud. Esta asociación también se ha encontrado en otros trabajos con reclusos ${ }^{28,42}$, y al igual que en uno de ellos no se observaron diferencias por sexo. Si bien en la población general se ha observado que las mujeres tienen una peor percepción de su salud que los varones ${ }^{41}$ no se aprecia tal diferencia en este estudio, aunque la desproporción del número de mujeres en esta muestra nos impide extender las conclusiones al respecto.

Hemos encontrado una asociación entre factores relativos a los tiempos de condena por cumplir y la salud percibida. Los que deben cumplir una condena mayor a cinco años, los que son reincidentes y los que no tienen juicios pendientes presentan una peor percepción de su estado de salud. Cuando incluimos en el modelo las variables de salud ser reincidente deja de ser significativo, lo que indica que dichas variables de salud son confundidoras en la relación entre la reincidencia y la percepción del estado de salud. Tras los análisis encontramos una mayor proporción de presencia de enfermedades crónicas entre los reincidentes con respecto a los que ingresan por primera vez, aún ajustando por edad (datos no presentados). No obstante, las variables de salud no interfieren en la asociación entre el tiempo de condena que tiene o está cumpliendo el recluso y la percepción de su salud. Este resultado podría estar indicando que la salud percibida se asociaría más que al tiempo real de estancia en prisión a la proyección que el recluso tiene del tiempo que tendrá que pasar en ella. Esta explicación también sería válida para los que no tienen juicio pendiente, es decir, para aquéllos en los que su suerte está echada. La imposibilidad de modificar las circunstancias o situaciones que son evaluadas como amenazadoras y destructivas lleva a las personas a un desánimo general, adoptando modalidades de afrontamiento poco adaptativas y rígidas que pueden asociarse a una mayor incidencia de morbilidad psíquica y física ${ }^{43,44}$. La situación de encarcelamiento es para las personas una situación arrolladora que escapa a su control, cuya prolongación en el tiempo la convierte en una fuente de ansiedad constante, mermando los recursos de afrontamiento, lo que puede ir en desmedro de su estado de salud.

El hecho de que los reclusos con alguna patología crónica o los que están tomando algún fármaco presenten una peor percepción de su estado de salud es esperable. Casi la mitad de los reclusos de la muestra manifiesta tener alguna enfermedad crónica. El porcentaje de personas que han declarado tener VIH $(19,1 \%)$ es similar al obtenido en otros estudios ${ }^{45} \mathrm{y}$ a las tasas de prevalencia obtenidas a partir de los registros de prisiones de un $16,6 \%$ en el año $2000^{4,11,46-48}$, y si bien en este trabajo no se tiene constancia de la infección por TBC algunos participantes han referido padecer la enfermedad $(2,7 \%)$, obteniéndose porcentajes coincidentes con otros trabajos ${ }^{49}$. Dentro de las variables asociadas a una mayor utilización de servicios sanitarios volvemos a encontrar el tiempo de condena que debe cumplir el recluso, existiendo un mayor uso por parte de aquéllos que tienen una condena mayor a cinco años. No obstante, aparentemente contradictorio, quienes llevan un año o menos en prisión utilizan en mayor medida los servicios sanitarios que los que llevan más de tres años. En otros trabajos ya citados se ha puesto de manifiesto una mayor utilización de servicios sanitarios por los reclusos conforme aumentaba el tiempo de encarcelamiento ${ }^{28}$. 
El hecho de que en esta muestra quienes llevan menos tiempo en prisión sean los que más utilizan los servicios sanitarios podría fundamentarse en que es al ingresar en prisión cuando se hacen las principales analíticas y diagnósticos de salud. También podría explicarse a través del consumo de drogas, puesto que gran parte de los participantes han declarado consumir heroína o sus derivados antes de entrar en prisión y más de la mitad consumían cocaína o crack. No obstante, estos porcentajes decaen notablemente tras el ingreso en prisión, por lo que tal vez los participantes acudan a los servicios sanitarios por consultas relacionadas con el cese del consumo de dichas substancias. Empero, en nuestro modelo de regresión ninguno de los consumos de drogas se ha mostrado relacionado con el uso de los servicios ni ha interactuado con la asociación encontrada con el tiempo de estancia en prisión. Esta explicación sobre la relación entre una menor estancia en prisión y una mayor utilización de servicios sanitarios podría apoyarse en el hecho de que la oferta existente en las prisiones de tratamiento para las drogodependencias podría estar por debajo de las necesidades reales, no accediendo, tal vez, a las mismas ofertas de tratamiento existentes fuera de la prisión ${ }^{50}$. Esto sugeriría una imperiosa necesidad de valorar y atender con mayor detenimiento y prontitud el estado de drogodependencia de presos a su llegada a la prisión. Es de notar que en este modelo, excepto el uso de fármacos (entre los que no se incluye la metadona), no se encuentran asociaciones entre las variables de salud y el uso de servicios sanitarios, como sería de esperar. Si bien en el análisis univariado, por ejemplo, el VIH o VHC se asocian a una mayor demanda de los servicios sanitarios no sucede lo mismo al introducirlos en el modelo multivariado. Es decir, en este modelo, quienes refieren padecer VIH o VHC no son los que más utilizan los servicios sanitarios de la prisión. Esto podría estar indicando que los servicios sanitarios carcelarios tras su labor de atención primaria (analíticas, vacunas) al ingreso en prisión, pondrían estar poniendo más énfasis en la dispensación de medicamentos y sustitutivos que en el seguimiento del progreso de posibles enfermedades crónicas.

En el presente trabajo hemos podido conocer el estado de salud percibida y el uso de los recursos sanitarios de un grupo de personas privadas de libertad de una cárcel andaluza. Además hemos podido identificar algunos de los factores asociados a dichas variables, los cuales deberían ser considerados a la hora de plantear posibles mejoras en la atención sanitaria en las prisiones. De ellos se desprende que hay que prestar mayor atención al tratamiento de los problemas asociados al consumo de drogas, al seguimiento de la evolución de enfermedades crónicas y, por último, entender el encarcelamiento mismo como una situación que puede empeorar las condiciones de salud psíquica y física de las personas confinadas.

\section{AGRADECIMIENTOS}

Los autores agradecen las sugerencias del/la revisor/a anónimo/a de la Revista Española de Salud Pública que permitieron mejorar notablemente este manuscrito. Los autores agradecen al Dr. Emilio Sánchez Cantalejo sus comentarios sobre los modelos estadísticos.

\section{BIBLIOGRAFÍA}

1. Dirección General de Instituciones Penitenciarias. La gestión en cifras. Madrid: Ministerio del Interior; 2004.

2. Plan Nacional sobre Drogas. Informe 5. Madrid: Delegación del Gobierno para el Plan Nacional sobre Drogas; 2002.

3. Plan Nacional sobre Drogas. Informe 3. Madrid: Delegación del Gobierno para el Plan Nacional sobre Drogas;2000.

4. Subdirección General de Sanidad Penitenciaria. Informe sobre la asistencia penitenciara en prisión. 
Madrid: Ministerio del Interior. Dirección General de Instituciones Penitenciarias; 2001.

5. García-Guerrero J, Herrero A, Bedia M, Araujo R, Castellano JC. Resistencias primarias de VIH en una población penitenciaria. Estudio REPRICOVA-2. Enferm Infecc Microbiol Clin 2004; 22(1):29-31.

6. Ruiz Pérez I, Soto Blanco JM, Olry de Labry Lima A, Antón Basante JJ, Castro Recio JM, Girela E. Morbilidad psíquica y apoyo social en reclusos en tratamiento con antirretrovirales. Rev Clin Esp 2004; (en prensa).

7. Olry de Labry A, Plazaola J, Ruiz I et al. Estado clínico y psicosocial de reclusos en tratamiento con antirretrovirales. Rev Esp Sanid Penit 2003; 5:92100

8. Iñigo Martínez J , Arce Arnáez A, Chaves Sánchez F, Palenque Mataix E , Burgoa Arenales M. Patrones de transmisión de la tuberculosis en un area sanitaria de Madrid. Rev Esp Salud Publica 2003; 77(5):541-51.

9. Paredes J, Colomer C. Medio penitenciario, VIH/SIDA y promoción de la salud. Gac Sanit 1997; 11(1):45-7.

10. Paredes i Carbonella J, Colomer Revuelta C. Prevención del VIH/SIDA en el medio penitenciario: una experiencia de plainificación participativa. Gac Sanit 2001; 15(1):41-7.

11. Pérez González K, Domingo-Salvany A, Hartnoll R. Prevalencia de la infección por el virus de la inmunodeficiencia humana y conductas de riesgo en consumidores de opioides visitados en un servicio de urgencias. Gac Sanit 1999; 13(1):7-15.

12. Cayla JA. Sobre la promoción de la salud en relación al VIH en el medio penitenciario. Gac Sanit 1997; 11(1):47.

13. Martín Sánchez V, Alvarez Guisasola F, Alvarez Fernández JL, Martínez Cordero MB. Efectividad (accesibilidad y adherencia) de un programa de detección precoz de tuberculosis pulmonar en población reclusa. Gac Sanit 1994; 8(44):203-8.

14. Acibaro L, Liras JA, Iglesias F et al. Factores sociales de riesgo para la falta de cumplimiento terapéutico en pacientes con tuberculosis en Pontevedra. Gac Sanit 2004; 18(1):38-44

15. Grupo Noroeste para el estudio de la Hepatitis por Virus $\mathrm{C}$ en el medio penitenciario. Seroprevalencia de infección por virus $\mathrm{C}$ de la hepatitis en población reclusa del noroeste de España a su ingreso en prisión. Rev Esp Salud Publica 1998; 72(1):43-51.

16. Alvarez Rodriguez M, Godoy García P. Prevalencia de la infección tuberculosa y por el VIH en los usuarios de un programa de reducción de riesgos para usuarios de drogas por vía parenteral (UDVP). Rev Esp Salud Publica 1999; 73(3):375-81.

17. Ferraro KF, Farmer MM, Wybraniec JA. Health trajectories: long-term dynamics among black and white adults. J Health Soc Behav 1997; 38(1):3854.

18. Grupo castellano-leones para el estudio de infección VIH en prisiones. Estudio de casos de infección por el virus de la inmunodeficiencia humana en prisiones de Castilla y Leon. Rev Clin Esp 2001; 201(5):249-55.

19. Cour MI, Martín Cala M, Fernández Contreras ME et al. Prevalencia de anticuerpos frente a VIH en drogadictos y reclusos. Rev Clin Esp 1989; 184(6):326-9.

20. Teplin LA. The prevalence of severe mental disorder among male urban jail detainees: comparison with the Epidemiologic Catchment Area Program. Am J Public Health 1990; 80(6):663-9.

21. Cattell V. Poor people, poor places, and poor health: the mediating role of social networks and social capital. Soc Sci Med 2001; 52(10):1501-16.

22. Coumans M, Spreen M. Drug use and the role of homelessness in the process of marginalization. Subst Use Misuse 2003; 38(3-6):311-38

23. Galea S, Vlahov D. Social determinants and the health of drug users: socioeconomic status, homelessness, and incarceration. Public Health Rep 2002; 117 Suppl 1:S135-45

24. Santana P. Poverty, social exclusion and health in Portugal. Soc Sci Med 2002; 55(1):33-45.

25. Martin V, Cayla JA, Bolea A, de Paz JA. Evolución de la prevalencia de la infección por Mycobacterium tuberculosis en una población reclusa al ingreso en prisión entre 1991 y 1996. Med Clin (Barc) 1998; 111(1):11-6.

26. Health status of detained and incarcerated youths Council on Scientific Affairs. JAMA 1990; 263(7):987-91.

27. Colsher PL, Wallace RB, Loeffelholz PL, Sales M. Health status of older male prisoners: a comprehensive survey. Am J Public Health 1992; 82(6):881-4. 
28. Lindquist $\mathrm{CH}$, Lindquist $\mathrm{CA}$. Health behind bars: utilization and evaluation of medical care among jail inmates. J Community Health 1999; 24(4):285303.

29. Moryl N, Santiago Palma J, Kornick C et al. Pitfalls of opioid rotation: Substituting another opioid for methadone in patients with cancer pain. Pain 2002; 96(3):325-8

30. Griffin MM, Ryan JG, Briscoe VS, Shadle KM. Effects of incarceration on HIV-infected individuals. J Natl Med Assoc 1996; 88(10):639-44.

31. Beyrer C, Jittiwutikarn J, Teokul W et al. Drug use, increasing incarceration rates, and prison-associated HIV risks in Thailand. AIDS Behav 2003; 7(2):153-61

32. Estébanez Estébanez P, Colomo Gómez C, Zunzunegui Pastor MV et al. Cárceles y SIDA. Factores de riesgo de infección por el VIH en las cárceles de Madrid. Gac Sanit 1990; 4(18):100-5.

33. Ferraro KF, Kelley-Moore JA. Self-rated health and mortality among black and white adults: examining the dynamic evaluation thesis. J Gerontol B Psychol Sci Soc Sci 2001; 56(4):S195-205

34. Ferraro KF, Su YP. Physician-evaluated and selfreported morbidity for predicting disability. Am J Public Health 2000; 90(1):103-8.

35. Health in prisons project. Healthcare study of the Irish prison population. WHO. Disponible en: www.hipp-europe.org/resources/internal/irish-prisons/0030.htm

36. Miret C, Miro O, Pedrol E et al. Demanda de asistencia hospitalaria urgente de la población reclusa. An Med Interna 1995; 12(4):175-81.

37. Gaiter J, Doll LS. Improving HIV/AIDS prevention in prisons is good public health policy. Am J Public Health 1996; 86(9):1201-3.

38. Edwards S, Tenant-Flowers M, Buggy J et al. Issues in the management of prisoners infected with HIV-1: the King's College Hospital HIV prison service retrospective cohort study. BMJ 2001; 322(7283):398-9.

39. Birmingham L. Should prisoners have a say in prison health care? BMJ 1997; 315(7099):65-6.
40. Idler EL, Benyamini Y. Self-rated health and mortality: a review of twenty-seven community studies. J Health Soc Behav 1997; 38(1):21-37.

41. Instituto Nacional de Estadística. Encuesta Nacional de Salud: personas por sexo, edad y percepción del estado de salud. [Web Page]. 2004; Disponible en: http://www.ine.es. (Consultado 15 de abril de 2004)

42. Fazel S, Hope T, O’Donnell I, Piper M, Jacoby R Health of elderly male prisoners: worse than the general population, worse than younger prisoners. Age Ageing 2001; 30(5):403-7.

43. Stowell JR, Kiecolt-Glaser JK, Glaser R. Perceived stress and cellular immunity: when coping counts. J Behav Med 2001; 24(4):323-39.

44. Lutgendorf SK, Costanzo ES. Psychoneuroimmunology and health psychology: an integrative model. Brain Behav Immun 2003; 17(4):225-32.

45. Babudieri S, Starnini G, Brunetti B et al. [HIV and related infections in Italian penal institutions: epidemiological and health organization note]. Ann Ist Super Sanita 2003; 39(2):251-7.

46. Carrasco Rodríguez P, Sánchez-Cruz JJ. Comparación de los conocimientos y los comportamientos de riesgo sobre el SIDA de los internos VIH+ y VIH- de la prisión provincial de Granada. Gac Sanit 1996; 10(54):104-9.

47. Marco A, Guerrero RA, Rodriguez AM et al Características de los casos de SIDA detectados en una prisión de Barcelona (1991-1993). Aten Primaria $1995 ; 15(8): 487-90$.

48. Rotily M, Delorme C, Galinier A, Escaffre N, Moatti JP. [HIV risk behavior in prison and factors associated with reincarceration of injection drug users]. Presse Med 2000; 29(28):1549-56.

49. Sánchez Moreno A, Villena Martínez L, Castro Recio JM, Aguayo Canela M, Pujol de la Llave E. Estudio de tuberculosis en la prisión de Huelva. An Med Interna 1997; 14(12):607-10.

50. EMCDDA Annual report on the state of the drugs problem in the European Union. Treating drug users in prison, a critical area for health promotion and crime reduction policy. Drugs in Focus 2002. 